\title{
Religiöse Zweifel und politische Gewissheit.
}

\author{
Eine Annäherung an Eric Voegelins Bild der Reformation \\ Calvins
}

Johannes Corrodi Katzenstein

\section{I.}

In diesem Text möchte ich EricVoegelins (1901-1985) Sicht des reformatorischen Wirkens Johannes Calvins skizzieren. Dem Unterfangen scheinen sich zunächst keine besonderen Schwierigkeiten entgegenzustellen. Das einschlägige Material umfasst weniger als dreissig Seiten, und die wesentlichen Punkte werden in einem einzigen Paragraphen (\$8) vorgetragen, dem eine etwas längere Diskussion des Wirkens Martin Luthers (\$1-7) vorausgeht. Doch der Anschein trügt. Der Interpretation dieser Passagen stellen sich beträchtliche Hindernisse in den Weg.

Voegelins ausführlichste Diskussion der Reformation Luthers und Calvins erscheint in Band 4 seiner History of Political Ideas. Der Band trägt den Titel Renaissance and Reformation (Band 22 in den Collected Works). ${ }^{1}$ Die insgesamt achtbändige History of Political Ideas umfasst mehrere tausend Manuskriptseiten. Doch hier beginnen auch schon die Schwierigkeiten: Die vorliegende History ist zu Lebzeiten ihres Autors nicht im Druck erschienen. Bloss einzelne Auszüge waren in Umlauf. Nach mehr als einem Jahrzehnt brach Voegelin anfangs der 50er Jahre die Arbeit am geplanten Werk dann definitiv ab und verzichtete auf die bevorstehende Publikation. Der Grund war, dass die theoretischen Grundlagen des Projekts dessen Urheber scheinbar nicht mehr zu befriedigen vermochten. ${ }^{2}$ Warum?

E.Voegelin, History of Political Ideas (HPI), 8 Bde., (= Collected Works Bde. 19-26), Columbia/London, erschienen zwischen 1997 u. 1999. Einzelne Teile der History liegen mittlerweile in deutscher Übersetzung vor, so auch der Reformationsteil: Luther und Calvin. Die grosse Verwirrung, hg. v. P.J. Opitz, München 2011. Ein weiterer kurzer Abschnitt, der Calvin gewidmet ist, findet sich im Nachfolgeband der History (Bd. 5/CW 23), Religion and the Rise of Modernity, hg. v. J.L.Wiser, Columbia/London 1998, 46-50.

2 P.J. Opitz, Der sneuen Innerweltlichkeit auf der Spur. Studien zu Eric Voegelins History of Political Ideas und seiner Deutung der westlichen Moderne, Voegeliniana 
Die werkgeschichtliche Rekonstruktion der History und deren sachlich-theoretische Einordnung im Gesamtwerk Voegelins befindet sich immer noch in den Anfängen. ${ }^{3}$ Denn was zunächst als ein weiteres textbook srosser politischer Ideen und Epochen im Lehrbetrieb höherer Bildungseinrichtungen in Nordamerika konzipiert wurde, entpuppte sich im Nachhinein als >Fragment einer etwas anderen politischen Philosophie, deren Anspruch und Ausmass damals wie heute quer zu konventionellen Textgattungen und wissenspolitischen Demarkationslinien standen. Die Rubrik, unter die Voegelins ausgearbeiteter Denkansatz fällt, könnte man als Geschichtsphilosophie nach dem Ende der Geschichtsphilosophie bezeichnen. Voegelin ist heute vor allem für sein fünfbändiges Reifewerk Order $\mathcal{E}$ History bekannt - wo er denn bekannt ist. ${ }^{4}$ In diesem Werk findet sich auch einiges an Material aus der History wieder. Aber, das sei nur am Rande erwähnt, auch das magnum opus ist von einem deklarierten >Bruch zeichnet $^{5}$, und der letzte Band ist wesentlich unfertig geblieben.

Für das 'Scheitern` der History scheinen nun mehrere Gründe massgeblich gewesen zu sein. Der wohl wichtigste war, wie Voegelin in seinen Autobiographical Reflections sagt, dass "the conception of a history of ideas was an ideological deformation of reality. There were no ideas unless there were symbols of immediate experiences. ${ }^{6}$ Was steckt hinter dieser Negativeinschätzung von >Ideen<? Im Unterschied zu Symbolen und den in ihnen lebendig vermittelten Erfahrungen von Gott, Welt, Mensch und Gesellschaft lassen sich Ideen gemäss Voegelin logisch so arrangieren, dass sie mit scheinbar innerer Notwendigkeit auf einen im Wesentlichen bekannten Höhepunkt des (über-)menschlichen Daseins hinauslaufen. Dadurch verdecken sie jedoch die radikale Ungewissheit, der die menschliche Erfahrung von Gott, Welt, Mensch und Gesellschaft ausgesetzt ist und auf alle Zeiten ausgesetzt sein wird. Das Drei-Stadien-Gesetz des Positivismus des neunzehnten Jahrhunderts, demzufolge das menschliche Denken durch und durch wissenschaftlich werden sollte,

\footnotetext{
- Occasional Papers 80 (2011), 5.

3 Die derzeit ausführlichste Diskussion bietet die oben angeführte Studie des ehemaligen Schülers Voegelins Peter J. Opitz.

4 E.Voegelin, Order \& History (OH), 5 Bde., Columbia/London (CW Bde. 14-18). Dt.: Ordnung und Geschichte, Hg. v. M. Henningsen u.a., 10 Bde., München 20012004. Siehe auch: M. Henkel, Eric Voegelin zur Einführung, Hamburg 1998.

5 Voegelin selbst spricht von einem Bruch im Programm, dessen Natur und Reichweite aber nicht ganz einfach zu bestimmen ist. Cf. dazu B. Douglass, The Break in Voegelin's Programm, in: Political Science Reviewer 7 (1977), 1-21.

6 E.Voegelin, Autobiographical Reflections, Columbia/London 2006 (CW 34), 90.
} 
ist für Voegelin Ausdruck dieser Art von Deformation der Erfahrung ebenso wie die Präfiguration des `Gesetzes` in der Erwartung eines Dritten Reiches des Geistes im Bewusstsein des mittelalterlichen Mönches Joachim von Fiore. ${ }^{7}$

Ein verwandtes Denkmuster sieht Voegelin nun auch hinter der vorherrschenden Textgattung der politischen Philosophie, zu der seine History beitragen sollte. Meist beginnt sie mit der griechischen Philosophie und den Anfängen des `vernünftigen ‘ Denkens, um dann ohne Verzug in die saufgeklärte` Gegenwart des Betrachters fortzuschreiten. Hier, so stellt sich heraus, sind die Ursprünge des Denkens (nahezu) vollendet. Durch die intensivere Beschäftigung mit dem 'finsteren Mittelalter, hinter dem die christliche Antike, Israel und die kosmologischen Hochkulturen des Nahen Ostens standen, gelangt Voegelin nun aber zum Urteil, dass "[t]he pattern of a unilinear development of political ideas, from a supposed constitutionalism of Plato and Aristotle, through a dubious constitutionalism of the Middle Ages, into the splendid constitutionalism of the modern period, broke down. $\ll^{8}$

Aber nicht nur der moderne Fortschrittsglaube kommt zunehmend ins Visier der Kritik. Selbst die weniger offensichtlich teleologisch-triumphalistische Geschichtsauffassung, dass wenn schon nicht das letztgültige Ziel der geistig-politischen Entwicklung der Menschheit im demokratischen Liberalismus, Sozialismus, etc., bekannt war, dann doch wenigstens die begrifflichen Mittel und Prinzipien zur >objektiven Beschreibung und Analyse dieser historischen Entwicklung 'wissenschaftlich` feststünden, war noch in die Annahme einer privilegierten Perspektive des modernen Betrachters verstrickt. Im Gegensatz dazu glaubt Voegelin nun aber erkannt zu haben, dass die von ihm so genannte shistoriogenetischer Deformation von Geschichte, davon das moderne Vertrauen in eine historisch privilegierte Gesellschaftsordnung bzw. in die rationale Analyse des politischen Gesamtprozesses eine blosse Variante darstellen soll, bereits in den kosmologischen Imperien des Nahen Ostens greifbar wird. ${ }^{9}$ Diese Einsicht hat Folgen. Unverkennbar wird damit das moderne wissenschaftlich-kritische Denken in eine epochenübergreifende Dynamik eingeschrieben, deren Erhellung selber nicht mit bekannten >rationalen K Kategorien beschrieben und analysiert werden kann.

\footnotetext{
7 Zu A. Comte cf. HPI 8/CW 26, 161-250; zu J. von Fiore bes. CW 5, $50 f f$.

8 Voegelin, Autobiographical Reflections, 90.

9 Cf. bes. OH 4/CW 17, 108-166.
} 
Das geschichtshermeneutische Prinzip, das Voegelin im Licht dieser seiner Einsicht in die smillenniale Konstante einer immer wieder durchbrechenden historiogenetischen Fehldisposition des menschlichen Bewusstseins entgegensetzt, lautet demnach: "The order of history emerges from the history of order. $«^{10}$ Weder >die Ordnung der Geschichte noch die zur Beschreibung und Analyse historischer Epochen und ihrer gesellschaftlichen Ordnungen notwendigen Begriffe stehen demnach fest. Wie können diese aber (wieder)gewonnen werden? Die Antwort folgt im unmittelbar anschliessenden Satz: »Every society is burdened with the task, under its concrete conditions, of creating an order that will endow the fact of its existence with meaning in terms of ends divine and human. ${ }^{11}$ Das Studium der Antike und des Mittelalters hat offenkundige Spuren hinterlassen im Grundansatz Voegelins.

Nun möchte man intuitiv vielleicht entgegenhalten, dass unter den Bedingungen einer sich als säkular verstehenden westlichen Moderne die rends divine r von Gesellschaft gerade weggefallen sind, und das zitierte Verstehensprinzip deshalb seine Bedeutung verloren hat. Dagegen wendet Voegelin wiederum ein, dass ein Wegfallen des gesellschaftlichenTranszendenzbezugs unter modernen Bedingungen nur auf dem Hintergrund des genannten Referenzsystems überhaupt erst als solches benannt und analysiert werden kann. Die vermeintliche Substituierung des göttlichen Ordnungspols durch ein rautonom gewordenes menschliches Handeln setzt gerade voraus, dass diese beiden Dinge nicht sschon immer identisch waren. Dies $\mathrm{zu}$ glauben ist 'man unter den Bedingungen eines >rein innerweltlichen Denkens allerdings gezwungen. Dieser Umstand macht die Sache einer umfassenden Geschichtsphilosophie insofern komplexer, als nun die $\mathrm{zu}$ analysierenden gesellschaftspolitischen Ordnungen auf einer spirituellen Grundlage basieren, die die Struktur eines (unthematischen) Glaubens wider besseres Wissen hat.

Die Spannung zwischen göttlichem und menschlichen Ordnungspolen zieht sich für Voegelin demnach als Konstante durch den Geschichtsprozess hindurch. Daraus folgt wie gesagt nicht, dass die Differenzierung des menschlichen Bewusstseins in Spannung zum göttlichen Grund ein geradliniger Prozess darstellte. Jede historisch errungene Balance zwischen den Polen ist fragil. Das dynamische Spannungsgleichgewicht zwischen spirituellen und politischen Ordnungsmächten, welches man aus Voegelinscher Perspektive zugleich als Getriebe und Kompass des Geschichtsprozesses be-

${ }^{10}$ E.Voegelin, Israel and Revelation (OH 1/CW 14), Columbia/London, 2001, 19.

${ }^{11}$ Ebd. Kursiv hinzugefügt. 
zeichnen könnte, droht je nach geistiger und politischer Situation immer auf die eine oder andere Seite hin aufgelöst zu werden. Die Folgen davon können schrecklich sein, wie zuletzt die spolitischen Religionen $<$ des zwanzigsten Jahrhunderts gezeigt haben. ${ }^{12}$

Die ideologisch-totalitäre Entwicklungslinie, der die Übernahme der geistlichen Macht durch den sich schon in der Renaissance und zuvor anbahnenden Typ von säkularem Intellektuellen vorangeht, stellt für Voegelin aber nur eine Art von Entgleisung des Geschichtsprozesses dar. Die spiegelbildliche Gegenseite dieser Art von Deformation des menschlichen Bewusstseins besteht in der versuchten rabsoluten Trennung von Religiösem und Politischem, bzw. zwischen religio und ratio. Auch sie ist, etwa durch den katholischen Rechtsgelehrten und englischen Renaissance-Humanisten Thomas Morus $^{13}$, gedanklich lange vorweggenommen worden. Paradoxerweise oder auch nicht führen gemäss Voegelin beide Deformationen letztlich zur innerweltlichen Vergöttlichung der Gesellschaft.

\section{II.}

Damit ist der Kontext fürVoegelins Analyse von Calvins reformatorischem Wirken ausreichend erläutert. Der Anfahrtsweg zu dem im Titel angekündigten Thema war zwar lang, aber dennoch notwendig. Denn wie bereits angedeutet, geht es Voegelin weder um eine srein theologische noch um eine srein historische oder politischphilosophische Auseinandersetzung mit dem Reformationszeitalter. Obwohl noch nicht so deutlich wie später in Order $\&$ History, lässt sich nämlich bereits hier das Verhältnis zwischen politischer und spiritueller Ordnung einer Gesellschaft als >eigentliches` Thema seiner Diskussion ausmachen.

Welches Verhältnis der >ends human and divine kommt in Calvins Institutio Christianae Religionis gemäss Voegelin nun zum Ausdruck? Dass die Einschätzung dieses Verhältnisses negativ ausfallen wird, lässt sich schon an der Überschrift des Reformations-Teils von Band 4 der History ablesen, die da lautet: The Great Confusion I. ${ }^{14} \mathrm{Nach}$ Voegelins Urteil hat das Reformationszeitalter nicht einen einzigen

\footnotetext{
${ }_{12} \mathrm{Zu}$ Voegelins Begriff der spolitischen Religion $\mathrm{cf}$. E.Voegelin, Die politischen Religionen, München ${ }^{3} 2007$.

13 Voegelin, Renaissance and Reformation, 117.

14 The Great Confusion II ist im nachfolgenden Band 5, Voegelin, Religion and the Rise of Modernity, anzutreffen und umfasst das zweite Kapitel der Abteilung, in dem Luther und Calvin diskutiert werden.
} 
grossen >politischen $<$ Denker hervorgebracht. ${ }^{15}$ Damit ist zugleich ein paralleles Urteil über die >Theologie` Luthers und Calvins gefällt. Man mag sich vielleicht wundern, wie letzteres aus ersterem folgen soll. Genau hier aber liegt der springende Punkt. Oder etwas vorsichtiger formuliert: ein wichtiger Grund für die düstere Gesamteinschätzung des Werks und Wirkens dieser in bestimmter Hinsicht unbestreitbar höchst brillanten Figuren liegt darin, dass sie die politische Dimension ihrer Situation nicht als konstitutives Element einer sreformierten` Theologie berücksichtigt hatten. Das Gegenteil ist der Fall. Die neue, vermeintlich >ursprünglicheく Glaubenslehre scheint die politische Verfasstheit des menschlichen Daseins in eine srein` natürliche Sphäre abzudrängen - in eine Sphäre also, deren Existenz für Voegelin gerade eine schlechte theologische Fiktion darstellt. Oder wie der Kritiker in Bezug auf Luthers Anthropologie statuiert: "The deep incision in the nature of man between his soul and his bodily existence makes the members of the dichotomy highly movable against each other. $«{ }^{16}$ Dass Leib und Seele des (Christen-) menschen nunmehr scheinbar vollständig getrennte Wege gehen können, sieht Voegelin in Luthers Doktrin der Rechtfertigung des Menschen allein aus Glaube begründet (256). Damit ist der Weg zu einer srein natürlichen Psychologie des Menschen geebnet, die sich nicht länger am transzendenten summum bonum des Menschen orientieren kann und will, sondern die Bestimmung des so genannt natürlichen Menschen allein im Innerweltlichen sieht (ebd.).

Calvin nimmt Luthers Rechtfertigungslehre grundsätzlich positiv auf. Doch genau diese Lehre hatte für Voegelin den schon bestehenden Auflösungsprozess der fragilen Ordnungen geistlicher und weltlicher Art auf eine Weise beschleunigt, dass eine theopolitisch auswegslose Situation entstehen musste (268). Das traditionelle Band der Freundschaft zwischen Mensch und Gott (amicitia dei) hatte die Spannung der Existenz >zum göttlichen Grund grundsätzlich bejaht. Doch nun wird diese Spannung durch die neue Grundkonstellation von >Gesetz und Evangelium s systematisch verdrängt. ${ }^{17}$ Warum? Die Frage stellt sich:Wenn der Christ über dem Gesetz steht, warum dann

15 "There is practically nothing in the plethora of partisan literature that would merit attention as the attempt of a first-rate mind to come to grips with the problems of his age." (HPI 5,/CW 23,17)

${ }_{16}$ Voegelin, Renaissance and Reformation, 256. Fortan sind die Seitenangaben im Haupttext zu finden.

${ }_{17}$ Hier sollte vielleicht vermerkt werden, dass die Tatsache der konfessionell-theologisch zum Teil sehr unterschiedlichen Ausarbeitungen dieser spirituellen Grundkonstellation in lutherischen und reformierten Kreisen fürVoegelin keine systematischen Konsequenzen zeitigt, die sein Gesamtbild grundsätzlich erschüttern könnten. 
nicht das Gesetz in die eigenen Hände nehmen und eine sgerechte Ordnung herstellen? Luther konnte sich zwar über den gewalttätigen Ausbruch antinomisch-revolutionärer Kräfte noch schockiert zeigen, die durch die erwähnte Dialektik und den damit verbundenen Gedanken des Priestertums aller Gläubigen entfesselt wurden (239). Dem Reformator aus Genf stand eine solche Reaktion aber definitiv nicht mehr offen (CW 23, 47).

Die Frage ist jetzt: Wie kann der Weg zurück in die Kontinuität gesellschaftstragender Strukturen gefunden werden, nachdem sich das Schreckgespenst der Revolution unabänderlich materialisiert hat? Voegelin stellt diese Frage in den Kontext seiner Diskussion von Calvins Umgang mit der traditionellen Prädestinationslehre. Die folgenreiche Entstellung des Sinns dieser durchaus >orthodoxen Lehre zeigt sich für Voegelin darin, dass der Reformator deren Erläuterung in der letzten Ausgabe von 1559 seiner Institutio aus dem üblichen Zusammenhang der Gotteslehre herausgenommen und in den dritten Teil (über den Geist) gestellt hatte - an seine Diskussion der Rechtfertigung durch Glauben anschliessend. Der neue systematische Ort des Topos der Prädestination kann entsprechend als sthe cure of souls (CW 22, 270) bezeichnet werden. Anders gesagt stärkt diese Verschiebung eine Entwicklung, in der die persönliche Glaubensgewissheit bzw. die Selbstvergewisserung des Einzelnen in sseinem Glauben immer mehr an Gewicht gewinnt. ${ }^{18}$ Damit drängt sich umgekehrt aber auch die Neutralisierung bzw.Verdrängung des philosophischen und religiösen Zweifels am Geist des rreformierten Christentums durch sskeptischer und radikale Bewegungen als potenziertes Problem auf. Denn einerseits kommt die gläubige Gewissheit 'wahrer Christen nicht ohne die psychischen und sozialen Kontrastfiguren des `Heiden` oder `Häretikers` zustande, andererseits lebt sie zugleich aber auch von deren Verdrängung. ${ }^{19}$ In der Geschichte des Christentums ist dieses Phänomen wohl nichts Neues. Doch scheinen sich die geistigen Bedingungen, unter denen

\footnotetext{
${ }^{18}$ Voegelin weist an dieser Stelle nicht darauf hin, dass Calvin mit seiner Entscheidung, die Prädestinationslehre auf die Glaubens- bzw. Rechtfertigungslehre folgen zu lassen, selbst unter reformierten Theologen relativ isoliert da steht.

${ }^{19}$ Ein analoges Verhältnis lässt sich heute wohl immer öfter zwischen ssäkular-liberalen und religiös-fundamentalistischen Lebensorientierungen erblicken. Der Unterschied ist bloss der, dass dieses Verhältnis durch das Dual Religion vs. Nicht-Religion anstatt durch das Dual wahrer Glaube vs. Irrglaube bestimmt ist. Je nachdem, welche Orientierung die moralische Mehrheit für sich beanspruchen kann, wird der eine Pol mit einem Plus, der andere mit einem Minus versehen.
} 
das Phänomen seine spätere gesellschaftspolitische Breitenwirkung entfalten kann, hier erst so richtig zuzuspitzen. ${ }^{20}$

Voegelins Darstellung der Institutio als >eigentlich` ordnungspolitischer Traktat mag erstaunen, wenn man dieses Werk als igrosses System < der evangelischen Theologie zu betrachten gewohnt ist (273). Voegelin hält einer solchen Wahrnehmung jedoch seinen vollen Widerspruch entgegen. Calvin mag zwar überzeugt gewesen sein, dass die Heilige Schrift, wenn sie denn ehrlich interpretiert wird, in eine einzige Gesamtaussage oder Lehre mündet (272). Um sich an diese Vorgabe aber halten zu können, musste doch einiges an Textmaterial ausgeklammert werden. Der beschworene 'Schriftgehorsam` und die Etablierung einer festgefügten theologischen Doktrin gehen nur schwer zusammen. Aber Calvins juristische Fähigkeiten lassen keine Schwäche erkennen, wenn es um die Glättung potentieller Falten in der einen und verbindlichen Doktrin geht (275-276). Kommt hinzu, dass der einzig verfügbare philosophische Denkhorizont, der seiner Theologie eine ssystematischer Kohärenz hätte verleihen können, sscholastischer Natur war, und deshalb dem Verdacht ausgesetzt war (272).

Dennoch glaubt Voegelin, eine Gesamtintention oder sintelligible Sinneinheit in Calvins Institutio zu erkennen (272). Hier lohnt es sich, etwas aufuhrlicher zu zitieren: "The strength of the Institutes lies in its concentration of argument on the realization of a practical purpose; and this purpose we can describe as an attempt to overcome Luther's impasse of 1525. The disintegration of order had left the Lutheran civitas Dei - the remnant', as Calvin calls it - of the truly faithful in dispersion in the wilderness of the reprobate. What should be done about this remnant, and could anything be done about it?" (273)

Zwischen den theologischen Zeilen gelesen, ringt Calvin gemäss Voegelin also mit einem eminent gesellschaftspolitischen Problem. Sollten die swirklich Gläubigen isolierte Individuen ohne Gemeinschaftsleben und Institution sein? Waren sie genug, um kleine lokale Zellen zu bilden, oder sollten sie sich vom Grossleib der Christenheit abspalten und in eine sektiererische Existenz gedrängt werden? Keine dieser Optionen vermag Calvin zufriedenstellen (273). Luthers >Rest` sollte vielmehr zur ’herrschenden Klasse` einer

${ }^{20}$ Charles Taylor äussert sich sehr ähnlich wie Voegelin, wenn er auf die Versuche der Reformationszeit hinweist, den religiösen `Halbschatten`, in dem weite Teile der Bevölkerung lebten, in Licht und Schatten aufzuteilen. Cf. Ch. Taylor, The Future of the Religious Past, in: Religion. Beyond a Concept, hg. v. H. deVries, New York 2008, 178-244, hier 243 
universalen Kirche werden, die die etablierte römisch-katholische Kirche früher oder später ersetzen würde (273).

Der Duktus von Voegelins Argument lässt keinen Zweifel daran aufkommen, dass sich hier eine dunkle Wolke revolutionären Unheils in der Geschichte des Westens zusammenbraut. ${ }^{21}$ Die 'wahre Ordnung (Kirche) soll die ‘falsche` Ordnung (Scheinkirche) ablösen. Das tödliche Potential späterer Revolutionen, von denen Calvin selbst noch nichts ahnen konnte, wird dadurch nicht verringert, dass dieser, wie schon Luther vor ihm, das traditionelle Glaubensverständnis als fides caritate formata (durch Liebe geformter Glaube) zurückweist, oder besser, seiner originären Position beraubt. Gerade dieser so verstandene Glaube stellt, wie Voegelin aber schon weiter oben deutlich gemacht hatte (250), die eigentliche spirituelle raison d'être der westlichen Zivilisation dar. Dazu nochmals ein etwas längeres Zitat: "The relationship of amicitia is mutual; it cannot be forced through an élan of human passion but presupposes the love of God toward man, an act of grace through which the nature of man is heightened by a supernatural forma. The loving orientation of man toward God is possible only when the faith of man is formed through the prior love of God toward man. " (250) Gemäss Voegelin war es gerade die so verstandene amicitia Dei, die von der theopolitischen Ordnung der hellenisch-christlichen Antike bis ins späte lateinische Mittelalter zwischen göttlichem Gesetz und göttlicher Gnade vermittelte, und die dadurch auch noch das Niedrigste und Alltäglichste im Leben zum ersten Schritt im >Aufstieg zu Gott verwandeln konnte. Die trennscharfe Unterscheidung von Natur und Gnade hatte die Frage nach dem eigentlichen und einzigen Subjekt dieser `Liebe Gottes dann aber in eindeutig theozentrischer Richtung aufgelöst (284).

Damit war die Frage, ob der `natürliche` oder `politische` Mensch diese Liebe im Glauben irgendwie erwidern, oder sich von ihr auch nur berühren lassen kann, in nachdrücklich abschlägiger Weise beantwortet. Menschliche Liebe $\mathrm{zu}$ Gott ist für Calvin selbst nur ein 'Werk ' des Menschen. Was danach kommt, wurde weiter oben schon erwähnt: die unmögliche Selbstvergewisserung des natürlichen Subjekts in sseinem Glauben, der zugleich immer ausserhalb seiner eigenen Möglichkeiten liegt (Gnade). In gewissem Sinn bedeutet das Höchstmass der Spannung im Verhältnis von Immanenz und Transzendenz, wie es hier vorzuliegen scheint, zugleich auch die Aufösung der Spannung. Von dieser Situation ist der Schritt zum vorläufigen Ende der Geschichte bei Voegelin nicht mehr weit.

\footnotetext{
${ }^{21}$ Cf. HPI 5/CW 23, 20.
} 
Ist der Geist des Protestantismus als gesellschaftlich orientierender Kraft des Westens einmal erloschen - und dies wird als fait accompli hingestellt - bleibt nur noch die Selbstvergewisserung des postchristlichen Subjekts durch sein eigenes sinngebenden Handeln in einem ran sich sinn-losen Universum. Das Prinzip des vox populi, vox Dei hat dann kein (asymmetrisches) Interpretationsverhältnis mehr im Auge, sondern degeneriert - in der modernen Idee der Volkssouveränität - zur spannungslosen Identität. Rationaler Zweifel an einer so verstandenen, >rein menschlichen Ordnung ist dann für Voegelin auch nicht mehr möglich.

\section{III.}

Abschliessend stellt sich die Frage, wie Voegelins Darstellung und Analyse der Reformation - und Calvins im Besonderen - kritisch evaluiert werden kann.

An einigen Stellen, das muss vorweg gesagt werden, spricht Voegelin nicht mit der Stimme des wissenschaftlich-distanzierten Forschers als vielmehr mit der Stimme des überhitzten Intellektuellen, der sich in den Dienst eines militant-liberalen Toleranzgedankens gestellt hat. Meines Erachtens kann Voegelins Demontage der hagiographisch überformten Person Calvins auf dem Hintergrund raufklärerischer Legenden eines so brillianten politischen Drahtziehers wie moralisch-sittlich verkommenen Finsterlings bestenfalls von anekdotischem Interesse sein. Aber Voegelins Kritik lässt sich dennoch nicht durch die blosse Richtigstellung >der Fakten < beseite schieben. Im Gegenteil erscheint ein solcher `verspäteter neo-positivistischer Appell an die Fakten geradezu deplatziert, wenn man seiner grossangelegten philosophischen Kritik am damals vorherrschenden Dogma einer /wertneutralen das Geringste abgewinnen kann. Vielmehr muss man sagen, dass Voegelin mit seinem Spürsinn für die unauflösliche gegenseitige Durchdringung theologischer und politischer Dimensionen der christlichen Botschaft bei Paulus bis zu Luther und Calvin eine Spur gelegt hat, deren Aktualität auch und gerade heute durch sehr unterschiedliche Philosophen, Denkansätze und Debatten unterstrichen wird.

Unterstrichen sollte hier auch werden, dass Voegelin keineswegs auf der Suche nach Sündenböcken ist, die man für alle Übel in der (modernen) Geschichte des Westens verantwortlich machen könnte. Meine Fokussierung auf die Person Calvins mag vielleicht 
einen solchen Eindruck erweckt haben. Der Eindruck löst sich aber rasch auf, wenn Voegelins Diskussion der Reformation im weiteren Kontext gesehen wird. Denn wie der fünfte Band der History zeigt, sieht Voegelin die 'grosse< Reform als eine, wenngleich herausragende Episode in einer grösseren Entwicklung, deren Wurzeln bis ins späte Mittelalter und weiter bis in die christliche Antike und die biblischen Traditionen zurückverfolgt werden kann. In dieser Entwicklung treten spirituelle Grunderfahrungen und Symbole, deren begrifflich-theoretische Ausarbeitung, sowie politische Ereignisverläufe und unintendierte gesellschaftliche Nebeneffekte in ein untrennbares Wechselverhältnis zueinander. Deshalb müsste man eher von einem umgreifenden Verstrickungszusammenhang sprechen, der in den Werken bestimmter Autoren zwar greifbar wird, sich aber kaum diesen selbst verdankt.

So gesehen scheint einer der umstrittensten und der weiteren Klärung am meisten bedürftigen Punkte zu sein: Inwiefern lässt sich Werk und Wirken Calvins als Weiterführung und Vorwegnahme religiöser und säkularer Reform- bzw. Revolutionsversuche darstellen? Oder präziser gefragt: Inwiefern lässt sich sein Werk und Wirken als Ausdruck einer zunehmend massenwirksamen Verdrängungslogik verstehen, der gemäss das >Alte angeblich von alleine verschwindet und nur einer verschwindend geringen Beihilfe durch den revolutionären Aktivisten bedarf? Schon bei Calvin, und ganz bestimmt bei späteren Generationen, die sich direkt oder indirekt auf ihn berufen, scheint sich für Voegelin der explizit bekundete Wille zur Reform letztlich nicht mehr vom impliziten Willen zur Revolution unterscheiden zu lassen. ${ }^{22}$ Damit ist ein Punkt erreicht, an dem die Texte, die in dieser Tradition verfasst wurden, letztlich überhaupt nicht mehr beim Wort genommen, sondern nur noch gegen den theologischen Strich gelesen werden können.

Freilich wird Gottes Souveränität und die Notwendigkeit der Unterordnung unter Gottes offenbartes Gesetz im Calvinismus theologisch stark hervorgehoben. Dieser Punkt entgeht Voegelin natürlich nicht. Aber gemeint ist eben immer die Unterordnung unter das göttliche Gesetz, so wie Calvin oder der jeweilige Interpret dieses

\footnotetext{
${ }^{22}$ Die Frage muss zumindest aufgeworfen werden, ob das Werk und Wirken Calvins hier nicht viel zu stark auf dem Hintergrund `des` Calvinismus, und dieser auf dem Hintergrund 'des` englischen Puritanismus gesehen wird. Zum Beispiel kann in der jüngeren Forschung der von Voegelin als Analyst und Antagonist der puritanischen Bewegung geschätzte Richard Hooker mit überzeugenden Gründen in die Nähe der Theologie der Reformatoren gerückt werden. Cf. etwa W. J. Torrance Kirby, Richard Hooker's Doctrine of the Royal Supremacy, Leiden 1990; C.C. Simut, Richard Hooker and his Early Doctrine of Justification, Aldershot 2005.
} 
versteht oder verstanden haben will. Hier zeichnet sich für Voegelin die Dynamik eines intellektuellen Willens zur Macht ab, der das intellektuelle Gewissen hinter sich zurückgelassen hat, und in den nachfolgenden Jahrhunderten immer virulenter wird. So gesehen kann die grundsätzliche Bestreitung der Notwendigkeit eines ssubjektiven< Interpretationsaktes zur Feststellung des >objektiven` Gehalts dieses Gesetz durch gewisse reformierte TheologenVoegelins Hermeneutik des Verdachts nur noch zusätzliche Nahrung verschaffen. Etwas Ähnliches gilt auch hinsichtlich seiner Negativeinschätzung der veränderten Funktion der Prädestinationslehre im Kontext einer frühmodernen cure of souls. Wenn sich 'wahre Gläubige ihres eigenen Glaubens prinzipiell vergewissern können, so scheint damit unweigerlich auch ein sozialpsychologisches Kontrastverhältnis gesetzt zu sein. Auf theologisch-dogmatischer Ebene wird zwar jeder Spekulation entgegengetreten, wer >drinnen Aber darunter wird der identitätsstiftenden Herausbildung verhaltenstypischer Merkmale für das Vorliegen des 'wahren` Glaubens gerade Vorschub geleistet. An diesem Punkt greifen meines Erachtens auch neuere Versuche der Richtigstellung von Voegelins Calvin-Kritik, wie etwa die von W. Stevenson ${ }^{23}$, noch zu kurz. Stevenson weist mit Recht darauf hin, dass für Calvin das Ringen um den wahren Glauben immer auch von Zweifel begleitet ist. Glaube und Zweifel sind deshalb keine Gegensätze. ${ }^{24}$ Was aber nicht gesehen wird, ist dass auch noch der Zweifel als ssignum electionis` der scheinbaren Indifferenz des Unglaubens entgegengehalten werden kann.Voegelins Lektüre der Reformation und Calvins ist bestimmt einseitig, wie Stevenson und andere überzeugend dargelegt haben. Eine umfassende Richtigstellung, die sich auf demselben Reflexionsniveau wie Voegelins Kritik bewegt, steht damit aber immer noch aus.

- Dr. Johannes Corrodi Katzenstein ist wissenschaftlicher Mitarbeiter am Projekt "Vertrauen verstehen“ am Institut für Hermeneutik und Religionsphilosophie an der Universität Zürich.

${ }^{23}$ Cf. W.R. Stevenson, Jr., An Agnostic View of Voegelin's Gnostic Calvin, in:The Review of Politics 66, 3 (2004): 415-443, und die dort angegebene Literatur.

${ }^{24}$ Ebd., 436. 